\section{Case Reports in Oncology}

\title{
Weather the Cytokine Storm: A Report of Successful Management of a Colon Cancer Survivor and a Critically III Patient with COVID-19
}

\author{
Mansoor Khalid ${ }^{a} \quad$ Tarek Dernaika $^{a} \quad$ Lirin Jacob $^{a} \quad$ Pavan Annamaraju $^{b}$ \\ Achuta K. Guddati ${ }^{\mathrm{c}}$ \\ aMercy Hospital, Oklahoma City, OK, USA; bJohnston Memorial Hospital, Ballad Health \\ System, Abingdon, VA, USA; 'Division of Hematology/Oncology, Augusta University, \\ Augusta, GA, USA
}

\section{Keywords}

Cancer $\cdot$ COVID-19 $\cdot$ Cytokine $\cdot$ Tocilizumab $\cdot$ Virus

\begin{abstract}
Patients with novel corona virus infection (COVID-19) can develop acute respiratory failure secondary to acute respiratory distress syndrome. Cytokine storm is suggested as one of underlying mechanisms for the rapid clinical decline. Immunocompromised patients and cancer patients are at particular risk for poor outcomes due to COVID-19 infection. This case report describes the presentation and clinical course of a cancer survivor who became critically ill and required mechanical ventilation. The patient was treated with hydroxychloroquine, azithromycin, and ceftriaxone; however, he remained febrile, hypoxemic, continued to require full mechanical ventilator support and his chest X-ray showed increased bilateral infiltrates. The patient was treated with tocilizumab, after which he improved and was successfully extubated. This report illustrates a possible role of tocilizumab in management of cytokine storm in critically ill patients with COVID-19 infection.

(C) 2020 The Author(s) Published by S. Karger AG, Basel
\end{abstract}




\section{Case Reports in Oncology}

\section{Introduction}

Human coronaviruses (HCoVs) are large, enveloped, single-stranded RNA viruses divided into 4 groups: alpha, beta, delta, and gamma, out of which alpha and beta are known to cause infection in humans. Four different types of HCoVs (HCoV 229e, NL63, OC43, and HKU1) are endemic and cause $10-30 \%$ of upper respiratory infections in adults [1]. Historically, HCoVs caused mild disease until recently when severe cases of atypical pneumonia called severe acute respiratory syndrome (SARS) by a beta-HCoV called SARS-CoV in 2002 in China and several other countries was reported. In 2012, another beta-CoV called Middle East respiratory syndrome (MERS) in Saudi Arabia were reported to cause respiratory failure. On December 31st, 2019, a cluster of novel coronavirus-infected pneumonia cases were reported in Wuhan, Hubei Province, China. The first 4 cases were apparently traced back to a seafood market in Hunan raising suspicion of a zoonotic $\mathrm{HCoV}$ outbreak. The origins of this outbreak continue to be a matter of great research interest currently. These cases were identified by using a surveillance mechanism that was developed in 2003 during SARS outbreak for early detection of novel pathogens like 2019-n CoV [2]. This disease was named as coronavirus disease 2019 by World Health Organization (WHO) and SARS-CoV2 by the Coronavirus Study Group in February 2020 [3]. Currently, there is no definitive treatment available for infected patients. Presence of elevated inflammatory cytokines like interleukin-6 (IL-6) and elevated ferritin indicates that this virus leads to a hyperinflammatory state causing cytokine release syndrome (CRS) that might play an important role in underlying pathology of critically sick patients. Tocilizumab is a humanized monoclonal antibody against the IL-6 receptor (IL6R). Endogenous IL-6 is released by inflammatory stimuli and controls a variety of immunological responses. Inhibition of IL6R by tocilizumab leads to decreased production of cytokines as well as acutephase reactants. It was approved in 2017 by the FDA for treatment of CRS [3]. Here, we present a case with successful outcome after treatment with tocilizumab.

\section{Case Presentation}

A 44-year-old Caucasian gentleman with past medical history significant for stage 2 colon cancer treated with sigmoid resection not on chemotherapy, mild obesity, nonsmoker, no known sick contacts or exposure to COVID-19, no recent travel history and no other comorbidities, presented to the emergency department with a 4-day history of fevers, headache, fatigue, myalgia, dyspnea on exertion, nausea, and diarrhea. His vital signs on admission showed a blood pressure $162 / 89$, heart rate $100 \mathrm{bpm}$, respiratory rate $24 / \mathrm{min}$, temperature $99^{\circ} \mathrm{F}$, $\mathrm{SpO}_{2} 95 \%$ on room air. Physical exam showed mild respiratory distress with normal breath sounds, no wheezing, rales, or rhonchi. Rest of the physical exam was normal. Laboratory tests showed WBC count 8,800/ $\mu \mathrm{L}$, lymphopenia 4\%, elevated D-dimer 2.99, elevated LDH 607, elevated CRP 241.1, and procalcitonin 0.35. His renal function tests and electrolytes were within normal range. His troponin and BNP levels were also normal. Viral respiratory pathogen panel was negative for influenza A and influenza B. His sputum cultures, blood cultures, and urine cultures were all negative. COVID-19 test done on admission. CT scan of the chest with and without contrast on admission showed extensive bilateral ground glass opacities (Fig. 1). EKG showed normal sinus rhythm and an incomplete right bundle branch block.

Patient was admitted to the medical floor initially; however, within $6 \mathrm{~h}$ of admission, he became severely hypoxic, his oxygen requirements rapidly increased to $10 \mathrm{~L} / \mathrm{min}$ and he was transferred to ICU where he was intubated and was empirically treated for presumed COVID- 


\section{Case Reports in Oncology}

Case Rep Oncol 2020;13:754-759

DOI: 10.1159/000509507

www.karger.com/cro

(c) 2020 The Author(s). Published by S. Karger AG, Basel

Khalid et al.: Tocilizumab Use in COVID-19

19 infection and community-acquired pneumonia with hydroxychloroquine (HCQ), ceftriaxone, and azithromycin. He also required vasopressor support due to hypotension. Patient was monitored closely for QT prolongation with daily EKGs. He did not develop any arrhythmias or QT prolongation during hospitalization. Despite all supportive measures, he continued to remain febrile, failed spontaneous breathing trials due to severe agitation, and continued to require full mechanical ventilator support, and positive end expiratory pressure had to be increased to $10 \mathrm{~cm} \mathrm{H}_{2} \mathrm{O}$ with $\mathrm{FiO}_{2}$ of $60 \%$ due to worsening of hypoxia. His follow-up X-rays showed increased infiltrates bilaterally (Fig. 2A). Patient was diagnosed with acute respiratory distress syndrome (ARDS). COVID 19 testing sent on admission came back as positive. Due to lack of improvement and increasing pulmonary infiltrates on the chest X-ray, he was given a single dose of tocilizumab $400 \mathrm{mg}$ on day 10 of admission. Within $24 \mathrm{~h}$, patient's respiratory status and mental status improved, and he was weaned off mechanical ventilation. He also became afebrile. Follow-up chest X-ray showed improved pulmonary infiltrates (Fig. 2B). Lymphopenia resolved and lymphocyte count was $45 \%$ on discharge. He was discharged home next day in a stable condition and repeat testing for COVID-19 was negative twice.

\section{Discussion}

COVID-19 is a beta coronavirus, whose genome is very similar to bat coronavirus, suggesting bat as its natural host. It uses angiotensin-converting enzyme 2 (ACE2) receptor to spread through the respiratory tract. Strong evidence shows human to human transmission [3]. Fever, cough, fatigue, and gastrointestinal symptoms are commonly reported symptoms of COVID-19 disease. The average reported incubation period of COVID-19 is 6.4 days and ranges from 0-24 days [4]. People above 65 years of age, with underlying comorbidities like hypertension, diabetes, cardiovascular disease, and chronic obstructive pulmonary disease have increased susceptibility to severe disease. Clinical features commonly include cough, fever, chest tightness, dyspnea, and diarrhea. COVID-19 is a multisystem disease that mainly targets and injures the vascular endothelium [5]. COVID-19 is clinically diagnosed by using real-time polymerase chain reaction (PCR) to detect nucleic acid swabs collected from nasal, throat, or other respiratory tract samples. Laboratory findings of patients show lymphopenia with low CD4 and CD8 lymphocytes, elevated lactate dehydrogenase, prolonged prothrombin time, elevated D-dimer, high alanine transaminase, creatinine kinase, and C-reactive protein $[6,7]$. Immunologic studies showed elevated plasma levels of ferritin, increased tissue necrosis factor- $\alpha$, elevated IL-6, IL-7, granulocyte colony-stimulating factor, interferon- $\gamma$-inducible protein 10 [8].

Radiologic findings on CT scan and chest X-ray show ground glass opacities or bilateral patchy infiltrates. Elevated levels of cytokines in COVID-19 disease suggest a hyperimmune response that leads to increased severity of disease and development of ARDS in COVID-19 patients. CRS is a condition that is caused by an unchecked hyperactivated immune system releasing immense amounts of proinflammatory mediators. CRS plays a major role in pathogenesis of several diseases like ARDS, macrophage activation syndrome, and sepsis. CRS is also a known toxicity of chimeric antigen receptor (CAR)-T-cell therapy. It is possible that CRS is a major underlying mechanism that leads to high severity of disease in COVID-19 patients.

Due to absence of definitive antiviral treatment from randomized clinical trials, current management of COVID-19 is mainly to control the source of infection, reduce risk of transmission, early diagnosis, and isolation along with supportive treatment focused on symptomatic management of patients [6]. Currently, all treatments for COVID-19 are under investigation, 


\section{Case Reports in Oncology}

Case Rep Oncol 2020;13:754-759 DOI: 10.1159/000509507

Khalid et al.: Tocilizumab Use in COVID-19

and their outcomes are unknown in suspected or confirmed cases of COVID-19. There are at least 291 clinical trials investigating pharmacologic therapy for adult patients with COVID-19 [9]. HCQ and azithromycin were used in a clinical trial to manage patients with COVID-19 [10]. HCQ and chloroquine are thought to inhibit binding of ACE2 receptors on host cell with the spike protein on the surface of beta coronavirus [11]. Despite its widespread use, so far there is lack of strong evidence to support the role of HCQ or chloroquine in improving outcomes [9]. Patients with refractory hypoxemia were recommended extracorporeal membrane oxygenation by WHO [12], convalescent plasma and immunoglobulin G are also being used in various clinical trials as potential treatment options for critically ill patients [13]. The rationale behind using this treatment is that antibodies from patients who have recovered from COVID-19 infection can probably help in clearance of free virus as well as infected cells [9].

On May 1, 2020, the Food and Drug Administration (FDA) approved emergency use authorization of a broad-spectrum antiviral medication - remdesivir for treatment of hospitalized patients with severe disease due to COVID-19. Preliminary results of Adaptive COVID-19 Treatment Trial (ACTT), showed 31\% faster time to recovery in patients who received remdesivir compared to those who received placebo. The group receiving remdesivir was found to have low mortality rate of $8.0 \%$ versus $11.6 \%(p<0.001)$ for the placebo group $(p=$ 0.059 ) [14]. Another study done in China by Li et al. [15] and published in the journal Lancet showed that remdesivir given to severe COVID-19 patients did not show any meaningful improvement in outcomes. The role of corticosteroids in treatment of COVID-19 patients is unclear. During the epidemics of SARS and MERS, observation studies did not show improved survival with corticosteroids; on the other hand, corticosteroids were associated with delayed viral clearance from respiratory tract and blood along with side effects including psychosis, hyperglycemia, and vascular necrosis $[16,17]$. Therefore, corticosteroids should not be routinely used in COVID-19 patients unless patients are in refractory shock or have chronic obstructive pulmonary disease exacerbation due to known side effects and lack of proven benefit [9].

Monoclonal antibodies that target inflammatory cytokines may have a potential role as an adjunctive treatment for patients with severe COVID-19 disease. Tocilizumab, a humanized monoclonal antibody is used for treatment of moderate to severe rheumatoid arthritis, giant cell arteritis, polyarticular juvenile idiopathic arthritis, systemic juvenile idiopathic arthritis, and treatment of CRS in adult and pediatric patients with CAR T-cell-induced severe or lifethreatening CRS. Common side effects of tocilizumab include upper respiratory tract infection, nasopharyngitis, headache, hypertension, increased liver enzymes, and injection site reactions [18]. Immunological findings in patients with severe COVID-19 revealed large amounts of IL- 6 secreted by pathogenic T cells and inflammatory monocytes that bind to IL6R on target cells, to develop cytokine storm leading to hyperinflammatory response in lungs and other organs causing severe disease [19]. Tocilizumab inhibits binding of IL-6 to IL6R and therefore can help prevent cytokine storm, thereby reducing immune-mediated damage to target cells [20]. In one study, 21 patients with severe COVID-19 disease showed immediate clinical and radiologic improvement after being treated with tocilizumab [21].

In this case report, we describe the successful treatment of a cancer survivor with severe COVID-19 with a single dose of tocilizumab. The patient became afebrile within $24 \mathrm{~h}$ of administration of first dose of tocilizumab; hypoxemia and delirium resolved, and he was successfully extubated. More interestingly, repeat PCR testing for COVID-19 was negative twice within 1 week of discharge from hospital. 


\section{Case Reports in Oncology}

\section{Statement of Ethics}

The patient had consented for his clinical history and case details to be published.

\section{Disclosure Statement}

No conflicts of interest exist for all the authors.

\section{Funding Sources}

No funding sources reported.

\section{Author Contributions}

Mansoor Khalid: literature review; wrote manuscript, revised manuscript. Tarek Dernaika: literature review; revised manuscript. Lirin Jacob: literature review; revised manuscript. Pavan Annamaraju: literature review; wrote manuscript, revised manuscript. Achuta K. Guddati: literature review; wrote manuscript, revised manuscript.

\section{References}

1 Paules CI, Marston HD, Fauci AS. Coronavirus Infections-More Than Just the Common Cold. JAMA. 2020 Jan;323(8):707.

2 Li Q, Guan X, Wu P, Wang X, Zhou L, Tong Y, et al. Early Transmission Dynamics in Wuhan, China, of Novel Coronavirus-Infected Pneumonia. N Engl J Med. 2020 Mar;382(13):1199-207.

3 Guo YR, Cao QD, Hong ZS, Tan YY, Chen SD, Jin HJ, et al. The origin, transmission and clinical therapies on coronavirus disease 2019 (COVID-19) outbreak - an update on the status. Mil Med Res. 2020 Mar;7(1):11.

4 Wang Y, Wang Y, Chen Y, Qin Q. Unique epidemiological and clinical features of the emerging 2019 novel coronavirus pneumonia (COVID-19) implicate special control measures. J Med Virol. 2020 Jun;92(6):568-76.

5 Greenland JR, Michelow MD, Wang L, London MJ. COVID-19 Infection: Implications for Perioperative and Critical Care Physicians. Anesthesiology. 2020 Jun;132(6):1346-61.

6 Wang D, Hu B, Hu C, Zhu F, Liu X, Zhang J, et al. Clinical Characteristics of 138 Hospitalized Patients With 2019 Novel Coronavirus-Infected Pneumonia in Wuhan, China. JAMA. 2020 Feb;323(11):1061-1069.

7 Huang C, Wang Y, Li X, Ren L, Zhao J, Hu Y, et al. Clinical features of patients infected with 2019 novel coronavirus in Wuhan, China. Lancet. 2020 Feb;395(10223):497-506.

8 Mehta P, McAuley DF, Brown M, Sanchez E, Tattersall RS, Manson JJ; HLH Across Speciality Collaboration, UK. COVID-19: consider cytokine storm syndromes and immunosuppression. Lancet. 2020 Mar;395(10229):1033-4.

9 Sanders JM, Monogue ML, Jodlowski TZ, Cutrell JB. Pharmacologic Treatments for Coronavirus Disease 2019 (COVID-19): A Review. JAMA. 2020 Apr. https://doi.org/10.1001/jama.2020.6019.

10 Gautret P, Lagier JC, Parola P, Hoang VT, Meddeb L, Mailhe M, et al. Hydroxychloroquine and azithromycin as a treatment of COVID-19: results of an open-label non-randomized clinical trial. Int J Antimicrob Agents. 2020 Mar 20:105949.

11 Zhou D, Dai SM, Tong Q. COVID-19: a recommendation to examine the effect of hydroxychloroquine in preventing infection and progression. J Antimicrob Chemother. 2020 Mar;dkaa114.

12 World Health Organization. Clinical management of severe acute respiratory infection when novel coronavirus infection is suspected. Geneva: World Health Organization; 2020.

13 Chen L, Xiong J, Bao L, Shi Y. Convalescent plasma as a potential therapy for COVID-19. Lancet Infect Dis. 2020 Apr;20(4):398-400.

14 National Institutes of Health. NIH clinical trial shows Remdesivir accelerates recovery from advanced COVID-19. Bethesda: National Institutes of Health; 2020. 


\section{Case Reports in Oncology}

\begin{tabular}{l|l}
\hline Case Rep Oncol 2020;13:754-759 \\
\hline DOI: 10.1159/000509507 & $\begin{array}{l}\text { @ 2020 The Author(s). Published by S. Karger AG, Basel } \\
\text { www.karger.com/cro }\end{array}$ \\
\hline
\end{tabular}

Khalid et al.: Tocilizumab Use in COVID-19

15 Li Z, Wang X, Cao D, Sun R, Li C, Li G. Rapid review for the anti-coronavirus effect of remdesivir. Drug Discov Ther. 2020;14(2):73-6.

16 Stockman LJ, Bellamy R, Garner P. SARS: systematic review of treatment effects. PLoS Med. 2006 Sep;3(9):e343.

17 Arabi YM, Mandourah Y, Al-Hameed F, Sindi AA, Almekhlafi GA, Hussein MA, et al.; Saudi Critical Care Trial Group. Corticosteroid Therapy for Critically Ill Patients with Middle East Respiratory Syndrome. Am J Respir Crit Care Med. 2018 Mar;197(6):757-67.

18 Sheppard M, Laskou F, Stapleton PP, Hadavi S, Dasgupta B. Tocilizumab (Actemra). Hum Vaccin Immunother. 2017 Sep;13(9):1972-88.

19 Fu B, Xu X, Wei H. Why tocilizumab could be an effective treatment for severe COVID-19? J Transl Med. 2020 Apr;18(1):164.

20 Sebba A. Tocilizumab: the first interleukin-6-receptor inhibitor. Am J Health Syst Pharm. 2008 Aug;65(15):1413-8.

21 Xu X, Han M, Li T, Sun W, Wang D, Fu B, et al. Effective treatment of severe COVID-19 patients with tocilizumab. Proc Natl Acad Sci USA. 2020 May;117(20):10970-5.

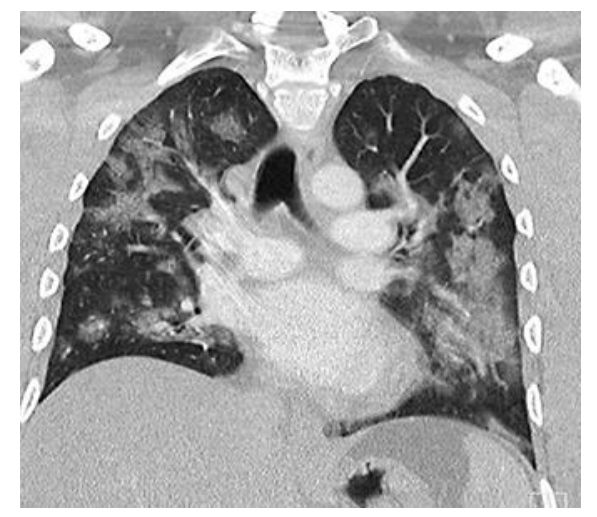

Fig. 1. CT scan of the chest on admission.
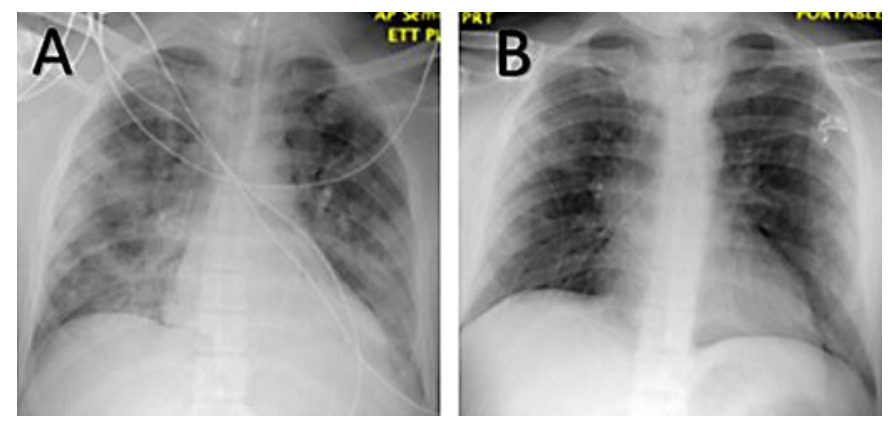

Fig. 2. Chest X-ray before (A) and after (B) tocilizumab administration. 